\title{
Translation as Co-creation with the Author
}

\section{Leilei Chen \\ University of Alberta}

When Ruth DyckFehderau-my colleague in the Department of English and Film Studies who was also my mentor for the Borderlines Writers Circle Program in 2017-2018-told me that she had just published a collection of short nonfiction stories about (and commissioned by) the James Bay Cree, I couldn't wait to read the book. I came to Canada in 2004 and later became a citizen, yet the country's First Nations always remained a mystery to me. The limited number of poems and stories I taught here and there in junior English literature courses, and the museums I visited where the indigenous peoples were mostly portrayed as "prehistoric," urged me to learn more about Canada's colonial past. The Sweet Bloods of Eeyou Istchee: Stories of Diabetes and the James Bay Cree contains real-life narratives told to the writer by people living on that territory. This "Talking Circle in print" (n.p.) as DyckFehderau calls it in her translation permission letter, was bound to satisfy my intellectual curiosity. And it did.

In particular, the aesthetics of "The Story of Maggie Happyjack and Simon Etapp of Waswanipi" piqued my interest and made me want to translate it into Chinese. Maggie and Simon's story-like others in The Sweet Bloods collection-does not have an exciting plot. Instead, the calm, steady unfolding of the life stories of Simon and Maggie reminds me of the slow, rhythmic sound of spinning yarn. Its peaceful tone- contrasted by the violence of residential schools and the tragic diabetic aftermath—renders the story strangely powerful. I hope Chinese language readers will feel the same aesthetic complexity in my translation. In the future I would like to translate the whole book, as I believe Chinese Canadians would benefit from knowing stories that subvert and complicate the pejorative stereotypes about First Nations that many Canadians falsely assume to be true. This includes Chinese-language readers in China and around the world. Translating The Sweet Bloods has become, for me, an emotional and ethical imperative.

Through the process of translating "The Story of Maggie Happyjack and Simon Etapp of Waswanipi," I witnessed the creative nature of literary translation which seems yet to be fully recognized. The theory of translation ethics emphasizes loyalty to the source text (Dingwaney 72; Venuti 17; Chen 64), but in reality, the translator must make the target text appeal to its readers in order to give it new life. The vitality of the translated version depends on its readability - its tactful and thoughtful breaking free from the confines of the linguistic structure of the original. My translation of the story aims to achieve this artistic goal while still maintaining "the integrity of the source culture" (Morgenstern-Clarren, n.p.). In the end, I feel as though I were the author of a Chinese story that happens to be entitled, "Maggie and Simon". The words are mine, but the rhythms belong to DyckFehderau. It’s “a kind of co-creation” (Moore, n.p.). 


\section{REFERENCES}

Chen, Leilei. Re-Orienting China: Travel Writing and Cross-cultural Understanding. Regina: University of Regina Press, 2016.

Dingwaney, Anuradha. "Introduction: Translating 'Third World' Cultures.” In Between Languages and Cultures: Translation and Cross-Cultural Texts, edited by Anuradha Dingwaney and Carol Maier. U of Pittsburgh P, 1995. 3-15.

Dyck Fehderau, Ruth. “Translation Permission” (personal document). 9 October 2019.

Moore, Jessica. "The Art of Translation.” Canadianart. 23 July 2018. https:// canadianart.ca/essays/the-art-of-translation/. Accessed on 6 February 2020.

Morgenstern-Clarren, Rachel. “The Translator Relay: Bill Johnston.” In Words without Borders. https://www.wordswithoutborders.org/dispatches/article/the-translator-relay-bill-johnston. Accessed on 6 February 2020.

Spivak, Gayatri Chakravorty. "The Politics of Translation." In Outside in the Teaching Machine, 179-200. London and New York: Routledge, 1993.

---. "Translating into English." In Nation, Language, and the Ethics of Translation, edited by Sandra Bermann and Michael Wood, 93-110. Princeton and Oxford: Princeton University Press, 2005.

Venuti, Lawrence. The Translator's Invisibility: A History of Translation. London and New York: Routledge, 2002. 


\section{Chinese translation of "The Story of Maggie Happyjack and Simon Etapp of Waswanipi" in The Sweet Bloods of Eeyou Istchee: Stories of Diabetes and the James Bay Cree}

瓦城玛姬和西蒙的故事

这是玛姬和西蒙两个人的故事，他们是魁北克省的瓦斯瓦尼皮（Waswanipi）小城 镇人。每当空气潮湿、日头高上的时候, 玛姬和西蒙的耳朵就有感觉, 就在他们耳膜曾经 破损又痊愈的地方，他们知道天气娘娘要说，今晚有低气压云层移到詹姆斯湾（James Bay）上空，该为这种天气做准备了。

西蒙比玛姬年长, 故事就先由他开始吧。他在米斯塔西尼（Mistissini）城外的树丛 中降生的时候就有心脏病。医生说他长大以后就会好的, 他后来确实好了, 但小时候病情 很重, 他的爸爸妈妈和其他照看他的人有时发现他倒在地上，不省人事，就知道他的心脏 病又发作了。西蒙心脏的情况很糟糕，他父亲认为他活不到成年。尽管如此，印第安事务 局的人还是在西蒙很小的时候，就把他从家里带走，送到苏圣玛丽（Sault Ste. Marie）的新 沃克（Shingwauk）寄宿学校。他那时候连鞋带都系不好，连学校都又把他送回家去，说 他太小了，他们不能收。（他后来不记得这段事了，是别人告诉他的。）不幸的是，印第 安事务局又径直把他送回了学校。

对这么年幼的男孩来说，新沃克是个可怕的地方。校舍在西蒙看来好像超级大。他 和其他的小男孩一起睡在一个摆着很多床、有着很高的窗户的长条形宿舍里。大点儿的孩 子睡在楼上。有个可怕的校长，一个叫伍兹的夫人，又老又凶，喜欢拿棍子打小孩；还有 一个金属质地、没怎么连接到地面的墙梯，供人在失火时逃跑，也让和西蒙一样年幼的孩 子们感到害怕。更糟糕的是, 西蒙的爸爸妈妈都病了。妈妈住进了医院, 爸爸的肩膀要手 术，医生已经订好了时间。远离疼爱他的人一一他们又在生病一一再加上待在一个常常让 他觉得害怕的地方, 西蒙开始焦虑, 他也许永远都见不到爸爸妈妈了。

有一天，西蒙和小伙伴们在外面雪地里玩，从坡上往下滑。他滑出了轨道，一直滑 到树枝边上, 刮伤了脸。他离开小伙伴去到医务室, 那里的护士给他的脸部做了包扎，然 后让他回宿舍休息。就在那儿，在新沃克的宿舍里，他倒在床上昏了过去。

所有看到这种情景的人，都见过西蒙昏倒、不省人事、心脏病发作接二连三全在一 天之内发生, 但对西蒙来说, 这次不一样。他的心脏完全停止了跳动。灵魂离开了他的身 
体，在天花板上的灯泡周围漂浮，然后穿过天花板，穿出屋顶，又飞到云端。他在持续升 高时, 能看到下面的一切渐渐变小。一个天使在云彩上等着他, 光亮洁白, 张开双臂迎接 他。天使和蔼地望着他，又把他送回下界，透过云层，透过屋顶，透过天花板，送回到床 上, 回到他的躯体。

西蒙在床上坐起身。托盘里的食物在等着他。他不知道是谁送了饭过来, 他拿起叉 子, 开始吃了起来。

这次经历五十五年以来他谁都没有告诉过，连他父母都没有。

新沃克已让西蒙感到度日如年，可几年以后，印第安事务局又把他送到布兰特福德 （Brantford）的莫浩克（Mohawk）学院寄宿学校，那里在他看来更糟糕。所有的土著孩 子都知道布兰特福德糟糕透了。那是加拿大最老的一间安立甘宗（Anglican）寄宿学校， 那里的人一直迫害儿童, 天长日久已经熟能生巧, 很多去了布兰特福德的孩子都死在那 里，更多孩子进去以后就再也没回过家。莫浩克族的孩子从 19 世纪 20 年代起就被送去布 兰特福德，克里族还算幸运，一百多年以来大部分人都幸免于难。西蒙和他的弟弟、两个 妹妹是第一批被送到那里的不幸的克里人。

玛姬的故事就从这里开始。她也来自詹姆斯湾，来自瓦城区域。她几年后才真正结 识西蒙，但他俩都是“糊糊洞”（Mush Hole）这个人间地狱里的克里人。幸运的是他们都 活了下来，剩下的全都是不幸。

他们刚到时，发生的第一件事就是女孩和男孩被隔离。玛姬是和弟弟一起来的（几 年后，另一个弟弟也进来了），可是弟弟被安置在不同的宿舍，她无法和他取得联系。时 不时地，清一色的女孩在院子里排成一行，刚好清一色排成一行的男孩也在，那时候，她 寸能看弟弟一眼, 知道他还好, 然后动动手指, 不作声响地打个招呼。在西蒙看来, 如果 玛姬在那样的队列里看到的是他, 冲他这样打招呼实在是太冒险了。

他们来到后的第二件事是剪发：玛姬和其她每个女孩一样，都剪了同样的发式，她 弟弟和西蒙，还有所有其他的男孩，都被剃了光头。那时，玛姬还很健康，她刚在家里过 了一个夏天，家里的食物很充足。护士记下了她的身高和体重，快速地上下打量她一番。 玛姬通常不会再见到护士，也不需要别人为她的身体操心，但是到了春季她回家的时候一 一回到疼爱她的家人身边的时候, 她的体重下降很多, 人都快要饿死了。 
人们把布兰特福德莫浩克学院叫做“糊糊洞”，因为那里的食物太糟糕了。玛姬能从 门缝里看到老师吃的好饭好菜, 她能闻到黄油烤牛肉、土豆和四季豆的香味, 可是她和其 他孩子却被迫吃早已生了虫的稀饭。虫子大都是死的，和稀饭一起煮，偶尔一个命大的死 里逃生, 还能蠕动几下, 把稀饭的表层弄得鼓起来。饭里没有虫子的时候, 也看不出是什 么东西, 难以入口。即便里面的东西他们能认得出, 像土豆或玉米, 也是煮了又竟, 并和 其它东西搅和到一块儿, 直到煮成一锅让人难以下咽的糊糊。有时是他们明明知道不能吃 的动物身上的东西——比如上面还带着毛的猪皮一也在碗里漂着。有时候碗里的东西根 本不是食物, 可能是玉米梗或者蔬菜皮, 又或者是面糊糊里掺点儿像木屑一样的东西, 再 放点儿盐, 有点儿咸味。

而且，老师会居高临下地站在孩子面前，手背在身后，眼里带着恐吓，孩子们知 道, 要是他们不逼着自己把糊糊吃下去, 就会挨打, 而且这顿打会把他们打到地底下去, 和那里的所有其他的孩子埋在一起。可是，他们的身体却说不了谎，即便晚饭过后，玛姬 有时还会饿昏过去，因为糊糊没有任何营养，她的身体还处于饥饿状态。夏季的时候，玛 姬和其他孩子会回家, 有肉、鱼和蓝芷吃, 身体又会恢复健康。之后, 秋季又无法逆转 地、无情地回来了，他们又被拖回糊糊洞，被迫咽下难吃的稀饭，继续忍饥挨饿。

糊糊洞的有些孩子在临近年终时，会有护士给他们检查身体，不是因为他们病了一 一太多的孩子经常因为肺炎和麻疹死在那里，也没有护士管他们一一而是因为有些像西蒙 和玛姬这样的孩子, 挨打太多, 被送进医院养伤。一个花样又一花样的殴打, 是打破糊糊 洞单调生活的主要方式。孩子们几乎每个星期都会挨耳光或头部被重击，尤其是后脑勺， 他们看不见也躲不过。办公室旁边有个小屋子，专门用来打人，里面放着两种皮带，一种 细细的，像皮鞭；一种宽扁的，每打一下，就会在皮肤上留下和它一样形状的红印子。工 作人员会把一个孩子带进小屋，关上门，不让孩子逃跑，在孩子赤裸的皮肤上一遍又一遍 地抽打。西蒙被打，是因为辅导员认为他手上的一块黑皮肤是泥巴，以为他没洗手。玛姬 挨打, 是因为她说克里话——那还是她刚来到学校除了克里话还不会说其它语言的时候, 还有一次被打, 是因为她实在太饿了, 从隔壁邻居树上摘了一个茾果吃。至于性侵犯么, 那就是家常便饭。人人都知道这种事情一直在发生。有时，校长会把一个小男孩或小女孩 抱到自己腿上, 堂而皇之地猥琶。还有一个辅导员叫鲍易斯先生, 到了晚上, 他就在睡着 孩子的一行行床间走来走去，突然一个孩子就会尖叫起来，每个人都知道鲍易斯先生又干 坏事了, 又在对另一个孩子性侵。 
甚至还有集体侵犯和虐待的情况发生。多年前，一个年龄大点儿的莫浩克族学生， 在一个孩子被打致死的地板上，看到过斑斑血迹。还有的学生胳膊被绑到水管上或头顶的 横梁上，就这样一个个光着膀子挨打。玛姬和西蒙没有这样的经历，这是他们长大成人以 后听说的。

西蒙有次住院是他十一岁的时候。他出了学校的地界，一个又胖又老、长着大手的 辅导员——罗欧先生一一对着西蒙的头和耳朵就是一顿猛打。通常这种事情发生, 疼痛一 两天就会消退，但在接下来的几天，西蒙的耳朵和头越来越疼。感染发炎了。一个星期之 后，他被送进六国门诊（Six Nations Clinic），他的耳膜被打破了。

尽管西蒙伤势令人震惊，尽管门诊的医生在检查中看得出他明显被虐待，他们却什 么都没问，只是给他消炎，然后把他送到布兰特福德的一个市级医院。这家医院也没好到 哪里。那里的护士和医生没有和颜悦色，也没有好言好语。他们只看他的伤口，保证他吃 喝拉撒正常和睡得安稳, 仅此而已。他们又把他送到阿姆斯（Amos）的医院继续康复。 接着，是放暑假的时间了，西蒙就回家了。秋季开始，他又回到了糊糊洞。

住在糊糊洞这种地方最糟糕的就是，人因为被虐待并一刻不停地处于惊恐之中，有 时会忘记怎样善待他人。有时，他们甚至会用别人对待自己的方式去对待其他人。有些大 点儿年龄的莫浩克孩子在那里待久了, 经历了各种各样的痛苦折磨, 变成了欺负年幼孩子 的恶霸。如果新来的年龄小的孩子是克里族, 不是莫浩克族人, 以大欺小的情况就更加恶 劣。因此，糊糊洞里最需要关爱的、年龄最小的孩子，没法儿在其他学生那里找到庇护。

西蒙 10 岁进糊糊洞, 在那里一直待到 12 岁; 玛姬 5 岁进去, 也一直待到差不多 12 岁。那儿没什么是为他们准备的, 就连合适的课程也没有。謷过每一天就是为了等夏季到 来, 他们就能回家了。一些其他族裔的孩子就连回家也不允许，他们必须常年待在那里。 对这些孩子来说，人类历史上再没有什么时段比他们在糊糊洞里的岁月过得更缓慢。

多年以后, 到了 2010 年, 开始有故事和新闻报道, 说调查人员在布兰特福德莫浩 克学院发现了刑具, 学院里的行政人员买土, 掩盖原住居民孩子的集体墓地。幸存者对他 们听到的故事和看到的报道并不奇怪。每当一个孩子失踪，老师都说“哦，他逃跑了”，或 者“她奶奶来把她接回家了”。孩子们当然知道老师在说谎。他们当然知道, 学校绝对不会 允许奶奶把孩子接回家的。 
就算回家了, 日子也不好过。大部分克里族父母自己都在寄宿学校待过，他们多多 少少知道自己的孩子在里面是什么情况。玛姬的爸爸妈妈从她和弟弟身上的累累伤痕和瘦 骨嶙峋的身体知道他们饱受虐待。他们也知道，很多孩子死在糊糊洞，玛姬很有可能就是 下一具被埋在那里的尸体。每到八月，玛姬就看到他们满面愁容，他们会说“上学去吧”, 也会交代大点儿的孩子照看他们，但玛姬看得出，他们不想让她走。最终他们又有什么办 法呢? 他们怎么能斗得过来掠走他们孩子的印第安事务局呢? 孩子们离开以后，父母都极 度悲伤, 村子里总是因此一连数日一片死寂。他们得找个解脱的办法, 于是就找到酒。喝 酒管用。

西蒙家里在暑假的时候也遇到了麻烦。首先是他爸爸的脚痛。后来双脚发炎始终不 好。再后来爸爸的一条腿被截至膝盖。从那以后, 家里的气氛就变了。爸爸现在坐轮椅, 以前能做的事情, 现在不能再做了, 妈妈很不高兴, 再加上妈妈的健康状况也越来越糟 糕。家里弥漫着他们阴郁的情绪。

其实, 西蒙的爸爸妈妈都得了糖尿病。糖尿病侵蚀了他爸爸的双脚，也让他妈妈患 了心脏病, 并最终因此丧生。等到他们明白怎样做才可以改善病情时，已经太晚了，他们 已经病得太重了。多年以后, 西蒙对此无法释怀：他的父母没能及时了解所有的信息, 帮 助他们控制自己的糖尿病发展。

他爸爸用手工劳动应对生活的变故。他一直是个烹饪好手。双腿还健全的时候, 整 个村子的宴席都是他来做。现在他坐着轮椅做饭, 做很多人的饭, 和面做面饼、经过一道 道工序做烧鹅、最后把它放进烤炉里烧烤。他还开始做木雕。他的卧室里有一堆华木，他 用一个有弧度的刻刀把它们刻成动物、碗和其它东西。他的卧室成了工作室（西蒙的妹妹 总是不停地清扫木屑）。后来，他还把自己的雕刻作品放在一个工艺品店出售。

有一年，秋季来临，西蒙没有被带回糊糊洞，而是去了在拉图克（La Tuque）的一 间学校。那仍是一所寄宿学校, 仍旧是监狱, 和暗无天日的孤独, 但对西蒙来说, 那里比 莫浩克学院好多了，好得难以置信。拉图克才是一所真正的学校。孩子们有真正希望他们 学知识的老师授课。他们晚上做完作业、家务和运动以后, 10 点就寝, 而不是像西蒙在糊 糊洞时那样， 7 点钟就被锁进宿舍里。拉图克有真正为学生设计的活动，划独木舟、野 营、游泳、走路, 还有很多很多的冰球运动。冰球设备简随得可笑, 孩子们得合用冰球 棍, 冰球棍破得早就该当柴烧了。冰鞋的刀刃也被磨过太多次了, 冰刀几乎都磨没了。他 
们的冰球队还是很棒, 当地白人的球队甚至企图把他们从社区球队中剔除。他们的水平之 高，直到现在还有人赞不绝口。

西蒙在这样的环境中脱颖而出。1966 年, 他在各种不同的比赛中成绩优异, 赢得 “年度最佳运动员”称号。后来，1967 年，他再次获此殊荣。拉图克当然也是寄宿学校，那 里当然也有虐待发生，但没有像在布兰特福德那样频繁，情况也没有那么恶劣。那里当然 也很恐怖，尤其是年幼一点儿、还不太懂事的孩子更害怕。那里当然还有欺辱。但是，西 蒙在糊糊洞里学到了最厉害的欺辱手段, 他知道如何以牙还牙。

玛姬的情况也比以前好了。她被送去鲁安诺兰达（Rouyn-noranda）读中学。她还是 得和弟弟分开，和她所爱的家人分开，还得待在寄宿学校被当作二等公民对待，而且，经 过了那么多年的虐待和恐吓, 她常常会感到抑郁。但是, 鲁安诺兰达给她的, 不只是威胁 和暴力，还有足够的食物、合格的老师和真正的学习机会。她成绩很好，被录取到渥太华 一所好的专科学校, 学习一年半文秘专业。

玛姬和西蒙的经历是人们无法想象的。他们已把糊糊洞抛到脑后。

可是, 人真能把像糊糊洞这样的经历完全抛到脑后吗? 真有人能走出它的阴影吗? 光是身体上的伤害就需要几十年的时间恢复。玛姬和西蒙的耳膜受伤严重, 在后来的二十 年，他们每人都做过好几次手术，修复他们破损的耳膜。外科医生让他俩把过去的病历拿 给他们看，以了解他们小时候在六国诊所和布兰特福德医院接受治疗和用药的情况。玛姬 打电话给诊所时，办事人员说，没有她和西蒙在那里住院的记录，记录已被销毁了。她又 给布兰特福德打电话, 电话那边的女士说的是同样的话: 十年以前的记录都被销毁了, 没 有玛姬、西蒙和其他寄宿学校学生的病历了。

然而，西蒙在布兰特福德住院之后，还去过阿姆斯的一家医院；玛姬被送回糊糊洞 以前, 也还去过瓦多尔（val d'or）医院。阿姆斯和瓦多尔的医院都是服务克里族社区的, 他们不需要销毁罪证。他们那里有玛姬和西蒙需要的所有信息, 还有他们遭受虐待的记 录。

除此之外，还有精神上的创伤。玛姬和西蒙极力想把糊糊洞的记忆从他们的生活中 去除，但是那些记忆还是会来侵扰他们。玛姬在商店里或是在散步的时候，看到一个孩子 受到了什么人或事情的惊吓，就会好端端地突然回到糊糊洞的办公室，不仅回想起曾经受 过的侮辱, 而且重新感受到每一顿拳打, 每一个耳光, 每一阵鞭打。她的抑郁症从 12 岁 
开始以来，一直一遍又一遍地折磨她，有时完全将她吞噬，令她卧床不起。她却挺过来 了, 她想过自己想要的生活，不是那些虐待她的人给她的生活。她坚持去看心理医生，医 治她情感上的创伤。这年复一年, 需要很长的时间, 也需要很多的努力。有的时候, 甚至 现在，抑郁还会偷袭她，让她防范不及。

西蒙试图用酒和毒品抹去不堪的往事，并在锯木厂打工兼做其它零工，以维持他的 嗜好。玛姬和西蒙在这段时间时常在一起, 西蒙对玛姬说, 她和他在一起应该小心点儿一 一他有严重的酒瘾和毒瘾问题。玛姬太了解糊糊洞记忆的威力了, 太了解它如何占据人的 大脑。她依旧和他在一起, 照顾他, 让他逐渐减少对毒品和酒精的依赖, 头脑渐渐清醒。 这也是一个漫长、缓慢的过程。

西蒙已经有三年都没犯毒瘾了，但他在睡眠状态下还是会发作。他会全身麻木，大 汗淋漓, 把床单都湿透, 幻觉在头脑中快速变换, 头痛欲裂, 然后心跳又慢下来, 他又继 续入睡。一天夜里, 西蒙毒瘾发作, 他向曾经救助过他的造物主祈祷, 求主让他不要再受 毒瘾的折磨。他感到主触摸了他，这种感觉传遍他全身，让他想到了几年前在云端和他相 遇的那个天使。那是他最后一次全身大汗淋漓。从那以后, 西蒙开始更虔诚地对待他的信 仰。

后来, 西蒙其它的健康问题接踵而出。 20 世纪 80 年代末, 西蒙和一个朋友为争取 领地的拥有权奔走相告。他穿上雪地鞋, 在雪里吃力地行走, 他长这么大, 从来没有感觉 过那么口渴, 一点儿力气都没有了。他身体确实虚胖了一些, 可他还是运动员的体格, 身 体从来都是听使唤的。如今他得喝一罐又一罐的果汁来维持他的体能，他的朋友早已远远 地走在前面，等着他跟上来。西蒙再去瓦尔多医院检查身体时，向医生询问了这个情况， 医院做了一些检查, 然后告诉他, 他得了糖尿病。离开医院的时候, 西蒙从护士那里拿了 药, 护士草草地跟他说, 他要注意饮食、加强锻炼了。

十年前的一个夏天，玛姬、西蒙和他们的女儿、孙子孙女们去郊外度假。孙子湿疹 发得厉害, 玛姬便开车带他去城里的门诊就诊, 在那儿照看孩子的护士发现玛姬面色苍 白, 于是就给她验了血, 发现她的血红蛋白值是 36, 而正常应该是 120 左右。玛姬严重贫 血。门诊用救护车把她送到阿姆斯医院治疗，她的孙子和其他家属也跟着救护车来到阿姆 斯。她在医院里躺了三个星期, 他们给她注射铁, 以提高她的血红蛋白水平。在那不久, 医生发现，她需要做子宫切除手术。术后有一次检查身体时，玛姬知道她也得了糖尿病。 
她并不奇怪——她妈妈就是死于糖尿病的。在糊糊洞忍饥挨饿、受尽折磨了那么多年, 自 然影响了他们身体消化食物的能力。

玛姬和西蒙反反复复经受了饥饿的折磨，现在，他们也患上了夺去他们父母生命的 糖尿病。他们又朝思暮想地渴望食物。多少年前, 他们的父母吃整盘整盘的面饼和果酱, 一边吃一边说, 他们要是有什么办法能控制他们的糖尿病就好了; 从来没有人告诉他们面 饼和果酱正在加剧他们的病情。西蒙去就诊时, 护士没有给过他什么信息, 他就自己学一 一查书、去图书馆、参加糖尿病患者烹饪班，他在从事急救工作中，直接接触患者，也遇 到很多糖尿病病人。他患病十五年以后，玛姬也被确诊得了糖尿病。那时他对这种病已经 有所了解, 比他们的父母了解得更多, 他厌倦了生病, 和玛姬反复谈论这件事。他们不是 糊糊洞里束手待毕的孩子。他们能够掌控自己的饮食, 掌控自己的生活。他们能自救。 他俩开始每天徒步几公里, 很快他们就感觉到, 在户外比在室内跑道上行走, 对改 善他们的精神面貌和血糖水平更有帮助。西蒙做饭比较多, 他对家里的膳食进行彻底改 造, 杜绝吃让他们血糖升高的甜点、面饼或土豆, 增加传统打猎获取的肉类和鱼, 这些食 物令他们立刻感到精力充沛。吃宴席时，西蒙对食物更有选择，他吃驼鹿和河狸肉，但把 肉汤留在锅里，因为肉汤很稠，是用面粉勾兑的，会让他血糖升高。他用菠菜、蓝莓、亚 麻籽和桂皮做成思慕雪，早餐时喝。他吃整把的果仁，试着用他在书里看到的自然控制血 糖的办法——比如用生洋葱或桂皮或青柠檬榨汁, 用水或苹果醋加蜂蜜调匀饮用。（孙子 孙女们觉得他这种食物实验有点怪。）看玛姬偶尔吃一块带有葡萄干的面饼解悗，西蒙一 口都不想吃。血糖升高无法控制, 这样嘴巴一时快乐, 不值得。况且, 有什么能比撒了香 料的、在明火上烧烤的白鱼、再佐以调了橄榄油的绿色蔬菜和黄瓜更美味的食物呢? 只有 他们整日都在户外的林中、时刻处于运动状态的时候，西蒙才会享受一块面饼或甜点的乐 趣。玛姬也努力节食, 她认真地给孙子孙女讲关于糖尿病和健康饮食的知识, 她吃西蒙做 的饭，只是她对自己没那么严格，有时，一块面饼的乐趣什么都无法取代。

玛姬和西蒙已不再年轻。他们经历的创痛是多数人都无法想象、更无法忍受的。可 更残酷的事情还在后头。2004 年, 布兰特福德莫浩克学院寄宿学校的克里族幸存者租了一 部巴士, 重返布兰特福德。他们想重新回到那片被鲜血浸染的土地, 直面他们在那里的遭 遇。这是件非常难做的事情。 
有些人一路都在车上坐着, 最后到了目的地, 他们却无法下车走进去。还有的在汽 车开进糊糊洞的那一刻, 就病倒了。玛姬和西蒙下了车, 泪流满面, 心跳不止, 他们又一 次穿行在宿舍、寝室和饭厅之间。

每走到一处，一幕幕画面便如潮水般涌了回来。就在这里，玛姬被鞭打得奄奄一 息; 在那里, 西蒙被残酷地强暴; 在这里, 玛姬的耳朵被拳头打得流血; 在那里, 西蒙浑 身僵直的站着，吓得不能动弹。每一个角落，每一个走廊，都让他们想起那种孤独无助的 感觉，想起成百上千的孩子经受的相同的遭遇。学校后面砖墙上写着一个又一个糊糊洞学 生的名字，那都是西蒙认识的人的名字，是那些同样担惊受怕、备受折磨的孩子的名字。 “请帮帮我吧! ”一个孩子在墙上这样写道。几十年过去了, 字里行间绝望的呼喊仍响彻耳 边。西蒙看着, 感到心被撕裂, 多年来深埋心底的痛苦又苏醒过来。

车上有个人说，他恨透了那些在他还是个小男孩的时候就虐待过他的人。“我会把 这恨带进坟墓的, ”他说。玛姬和西蒙很理解。他们也恨。恨把西蒙逼得酗酒吸毒成瘾, 把他变成一个放纵惧落的人, 要面对一大堆的个人问题。也是恨导致了玛姬严重的忧郁 症。

但玛姬和西蒙不想把恨带进坟墓。他们回到学校是为了另一个原因。他们要原谅那 些做过伤天害理事情的人。不是因为那些施虐者想要他们原谅——他们蓄意销毁学校记 录，说明他们很可能毫无攸过之意。原谅对他们无济于事。玛姬和西蒙的原谅是为了他们 自己，为了他们能够痊愈，从而最终不在对糊糊洞耿耿于怀。

他们站在那个可怖之地，祈祷。先感谢造物主，让他们自己的旧伤痊䏒，接着他们 祈求主的原谅——原谅折磨他们的人, 也原谅他们自己学着用同样的欺凌手段伤害过其他 人。

几年之后，西蒙参加过六个原住居民族群幸存者的聚会，一些关于糊糊洞的老故事 又被说起。这一次, 这些往事对他的伤害没有那么深了, 西蒙知道, 他的祈䘠灵验了, 他 真正痊㸝了。

原谅是个奇怪的字眼, 它听起来好像是一件容易做的事，但有时却是最难的、的的 确确最难做的决定。有时作出原谅的决定需要你倾其所有。西蒙和玛姬都做到了。 
TranscUlturAl, vol. 12.2 (2020), 79-90.

http://ejournals.library.ualberta.ca/index.php/TC

这是关于魁北克省瓦斯瓦尼皮小城玛姬和西蒙两个人的故事。每当空气潮湿、日头 高上的时候, 玛姬和西蒙的耳朵就有感觉, 就在他们耳膜曾经破损又痊缌的地方, 他们知 道天气娘娘要说，今晚有低气压云层移到詹姆斯湾上空，该为这种天气做准备了。 